\title{
Percutaneous Transluminal Septal Alcoholization for the Treatment of Refractory Hypertrophic Obstructive Cardiomyopathy. Initial Experience in the Federal District
}

\author{
Evandro César Vidal O sterne, Tamer Najar Seixas, Walter Paulo Filho, \\ Ernesto Misael Cintra O sterne, O toni Moreira Gomes
}

Brasília, DF - Belo Horizonte, MG - Brazil

\begin{abstract}
Objective - To analyze the efficacy of percutaneous transluminal septal alcoholization in the treatment of refractoryobstructive hypertrophic cardiomyopathy (HOC).

Methods - The patients were referred for alcoholization after Doppler echocardiography. Before and after alcoholization, the intraventricular pressure gradient was recorded. Alcoholization was performed with a $3 m L$ injection of absolute alcohol through a coronary angioplasty balloon catheter. The procedure was concluded after a significant reduction or abolition of the pressure gradient.
\end{abstract}

Results - Of 22 patients, 18 (81.8\%) successfully concluded the procedure with a reduction in intraventricular pressure gradient at baseline (from $67.6 \pm 24.2 \mathrm{mmHg}$ to $3.8 \pm$ $1.9 \mathrm{mmHg}, p<0.005$ ) and after extrasystole (from $110.4 \pm$ $24.2 \mathrm{mmHg}$ to $9.6 \pm 2.6 \mathrm{~mm} \mathrm{Hg}, p<0.005)$. A significant reduction in mean interventricular septal thickness (from $2 \pm$ $0.3 \mathrm{~mm}$ to $1.7 \pm 0.2 \mathrm{~mm}, p<0.005)$ and in peak pressure gradient (from $90.7 \pm 23.5 \mathrm{mmHg}$ to $6.1 \pm 1.4 \mathrm{mmHg}, p<0.005$ ) was observed on Doppler echocardiography after 6 months, when all patients were in functional class I. The most frequent acute complication, present in $11 \%$ of the patients, was the need for definitive pacing implantation. Relapse of the symptoms and reappearance of the pressure gradient occurred in $16.6 \%$ of the patients. One patient (5.5\%) died probably due to a diffuse coronary spasm prior to the procedure, and another died suddenly on late follow-up.

Conclusion - Percutaneous transluminal septal alcoholization is effective and safe in the treatment of HOC.

Keywords: percutaneous septal alcoholization, percutaneous septal ablation, hypertrophic obstructive cardiomyopathy, ablation

Hospital das Forças Armadas (HFA), Instituto do Coração de Taguatinga - Brasília and Fundação Cardiovascular São Francisco de Assis - Belo Horizonte.

Mailing address: Evandro C. V. Osterne - SRES, Quadra 12, Bloco U - Casa 35 70645-215 - Brasília, DF - Brazil - E-mail: osterne@ambr.com.br English version by Stela Maris C. e Gandour
Hypertrophic cardiomyopathy is the most common genetic cardiac disease, and, in most cases, it has a dominant autosomal transmission pattern. From the anatomicopathological point of view, it is characterized by left ventricular hypertrophy, disorganization of the myocytes and myofibrils, an increase in collagen, and alterations in the intramyocardial coronary arteries, with no other cause of increase in the myocardial muscular mass. The enormous interest about the real incidence of hypertrophic cardiomyopathy and the development of methods capable of detecting its presence is because it is the major cause of sudden death among young people, mainly athletes ${ }^{1-4}$.

The studies published have reported a great variation in its incidence, which ranges from 0.2 to $5 \%$ of the population. More consistent studies, however, estimate approximately 2 cases per 1,000 inhabitants ${ }^{5,6}$.

The clinical course of hypertrophic cardiomyopathy is extremely variable, and the great majority of patients remain asymptomatic during their entire life. A minority, although a significant number of patients, has symptoms of heart failure, angina, and syncope, and some may die suddenly ${ }^{7-13}$. The treatment has varied from the use of antiarrhythmic drugs of the amiodarone type to the implantation of cardioverter-defibrillators ${ }^{14-19}$.

In regard to patients with the obstructive form of hypertrophic cardiomyopathy, characterized by the presence of an important dynamic intraventricular pressure gradient, approximately 5 to $10 \%$ are estimated to persist with disabling symptoms refractory to thorough pharmacological therapy or to high doses of medication, which cause important side effects similar to the symptoms of hypertrophic cardiomyopathy. For the latter, 2 alternatives of invasive treatment existed until recently: the surgical treatment with Morrow myotomy-myectomy with or without mitral valve replacement, and the electric treatment with implantation of dual-chamber pacemaker of the DDD type ${ }^{20-35}$.

The most recent alternative in invasive treatment for 
refractory hypertrophic obstructive cardiomyopathy appeared with Sigwart's original report ${ }^{36}$ on the reduction in septal mass after myocardial infarction induced by alcoholization of the septal artery. The target vessels were the first septal branches of the anterior interventricular artery, which, in most cases, is the major branch of the left coronary artery. These first branches are responsible for the irrigation of the abnormally hypertrophic proximal septum that causes obstruction of the left ventricular outflow tract.

Several studies carried out later confirmed the efficacy of the permanent interruption of the septal artery in cardiac catheterization laboratories, seen as a reduction in the intraventricular pressure gradient, in the abnormally hypertrophic septal thickness, and in symptomatology ${ }^{37-55}$.

Our study aimed at analyzing the efficacy of septal artery alcoholization in the treatment of refractory hypertrophic obstructive cardiomyopathy in patients treated and followed up at the Hospital das Forças Armadas and the Instituto do Coração de Taguatinga, in Brasilia, Federal District.

\section{Methods}

From August 1998 to February 2001, 54 patients with hypertrophic obstructive cardiomyopathy confirmed on Doppler echocardiography were referred for percutaneous transluminal septal alcoholization, 22 of whom were selected for the production of therapeutic septal infarction. The selection of patients was initially based on refractoriness to thorough clinical treatment defined as persistence of important symptomatology despite the use of propranolol or verapamil at the dosage of $240 \mathrm{mg} /$ day. Other patients were also selected due to symptoms, such as dyspnea, dizziness, and lipothymia even when taking low doses of those drugs, which caused the interruption of the medications. Two patients, who already had implanted pacemakers of the DDD type when referred, remained in NYHA functional class III or IV even with the concomitant use of beta-blockers and diuretics.

The hemodynamic inclusion criterion was the existence of an intraventricular pressure gradient $>60 \mathrm{mmHg}$ at baseline or after extrasystole. This level of pressure gradient (peak gradient) observed on Doppler echocardiography also helped to select the candidates for treatment. As an immediate success criterion, the residual gradient at baseline $<20 \mathrm{mmHg}$ was adopted.

All patients were followed up through monthly telephone contact and an ambulatory visit every 3 months. Doppler echocardiography was performed at the end of 6 months.

In the statistical study, the Student $t$ test was used to analyze the following independent variables: 1 ) obtained on Doppler echocardiography (before and 6 months after alcoholization): interventricular septal thickness, left ventricular posterior wall thickness, left atrial diameter, septum/posterior wall ratio, and peak pressure gradient; and 2) obtained through manometry in the catheterization room (immediately before and after alcoholization): pressure gradient at baseline and after extrasystole. The significance level (p) of the statistical test was fixed at $\mathrm{p}<0.05$ (confidence interval, CI $>$ 95\%).

Age ranged from 21 to 51 (mean of 39) years; $12(54.5 \%)$ patients were males and $10(45.5 \%)$ were females. Most patients alternated between NYHA functional classes III and IV (class III/IV, of chart I) on their ambulatory visits with full doses of medication.

The 22 patients chose the percutaneous procedure and also agreed to participate as research subjects signing the informed written consent, according to resolution 196 of the National Health Board of the Health Ministry, which regulates research involving human beings $\mathrm{s}^{55}$.

Chart I shows the major characteristics of the patients studied. All patients had previously undergone the following routine laboratory tests: complete hemogram, urea, creatinine, potassium, T3, T4, chest teleradiography, electrocardiography, and Doppler echocardiography. All patients also underwent sedation and analgesia, which were conducted by an anesthesiologist present in the procedure room, who used 1 or more of the following drugs: fentanyl, midazolam, sevoflurane, propofol, diazepam, and meperidine, at individualized dosages. The intravenous medication was administered in the vein of the left elbow fold. The electrocardiogram and digital $\mathrm{O}_{2}$ saturation were also monitored.

Two arterial and 1 venous access routes were used for the procedure itself, and most cases followed the routine as shown. After local analgesia with $2 \%$ lidocaine, the right brachial artery and vein were dissected, and the right femoral artery was punctured. An 8F-diameter plastic and valvulated sheath was positioned at the site of the arterial puncture. Through a retrograde arterial access route in the right arm, a Lehman or $8 \mathrm{~F}$ ( 8 French in diameter) Cournand

\begin{tabular}{|lc|}
\hline \multicolumn{2}{|c|}{ Chart I - Characteristics of the population studied } \\
\hline Variable & Condition \\
\hline Age & $21-51$ years \\
Sex: & 12 \\
Male & 10 \\
Female & 15 months \pm 7 \\
History & 7 \\
Familial history of sudden death & 2 \\
DDD-PM & 6 \\
Systemic arterial hypertension & 16 \\
Medication used: & 6 \\
Beta-blocker & 4 \\
Calcium antagonists & 6 \\
Previous complications: & 1 \\
Syncope & 11 \\
SVPT & 2 \\
Symptomatic bradycardia & 4 \\
CHF & \\
Atrial fibrillation & 3 \\
NYHA functional class: & \\
Class III & \\
Class III / IV & \\
Class IV & \\
\hline N - number; DDD-PM - dual-chamber pacemaker; SVPT - supraventricular \\
paroxysmal tachycardia; CHF - congestive heart failure; NYHA - functional \\
classification of the New York Heart Association (USA); class \\
patients alternating classes III and IV at each ambulatory visit. \\
\hline
\end{tabular}


catheter with a single orifice at the extremity was advanced and left in the left ventricular tip. Also through the retrograde arterial access route, beginning in the femoral artery, a guiding coronary angioplasty catheter was positioned in the left coronary ostium. This guiding catheter had lateral orifices, a premolded extremity for selective catheterization of the left coronary artery (JL4), and a diameter of 7F or 8F. A $6 \mathrm{~F}$-diameter bipolar electrode catheter was advanced through the intravenous access route from the right elbow fold to the right ventricular tip, and connected to a pulse generator, which was left on standby. All procedures were performed in the posteroanterior cranial projection, because it is the one that better visualizes the first septal branches of the anterior interventricular artery.

After administering 5,000U of sodium heparin, contrast medium was selectively injected in the left coronary ostium, and the first major septal branch of the anterior interventricular artery, the one chosen for the ischemia test, was visualized. Then a 0.014", 180-cm-long, extra-support, J-tip, metallic guidewire (Stabilizer PLUS, Stabilizer-XS - CordisUSA, and BMW-HiTorque-Guidant - USA) was advanced through the anterior interventricular artery. After rotatory and "push-and-pull" maneuvers, the chosen septal branch was entered, and the guidewire was advanced even further to leave its extremity as deep as possible within the vessel. Right afterwards, an over-the-wire balloon (Thor, Charger, Valor and Predator - Cordis - USA, and Ranger - Boston Scientific - USA), with an inflated diameter of 2.0 to $2.5 \mathrm{~mm}$ and a length not exceeding $20 \mathrm{~mm}$, was advanced within the septal branch and inflated until its nominal pressure was reached, usually around $6 \mathrm{~atm}$. The balloon was maintained as such for a maximum of 15 minutes.
Septal alcoholization in hypertrophic obstructive cardiomyopathy

During this period, the simultaneous LV/AO pressure reading and the behavior of the pressure gradient with ischemia caused by the inflated balloon were observed (figs. 1 and 2).

The significant drop in the pressure gradient with the provoked ischemia signaled the continuation of the procedure. The metallic guidewire was then withdrawn to inside the guiding catheter, leaving only the inflated balloon inside the septal branch. Right afterwards, a small amount of contrast medium was injected with the balloon, aiming at determining the size of the area irrigated by the septal branch and at verifying the existence of reflux to the anterior interventricular artery. The persistence of a significant pressure gradient after 15 minutes determines the choice of another septal branch for alcoholization.

Alcoholization was performed with a 3-mL injection of absolute alcohol through the inflated balloon catheter after deepening of analgesia by the anesthesiologist. Once alcohol infusion was finished, the balloon was maintained inflated for approximately 3 minutes. Then, its interior was washed with saline solution, and the balloon was deflated and withdrawn to inside the guiding catheter. After a pressure recording showing the absence of an intraventricular pressure gradient, new opacification of the left coronary artery was performed with contrast medium to confirm septal branch occlusion (fig. 3).

\section{Results}

Alcoholization of the first septal branch of the anterior interventricular artery or of the first major branch was successful in $16(88.8 \%)$ patients after occlusion of a single

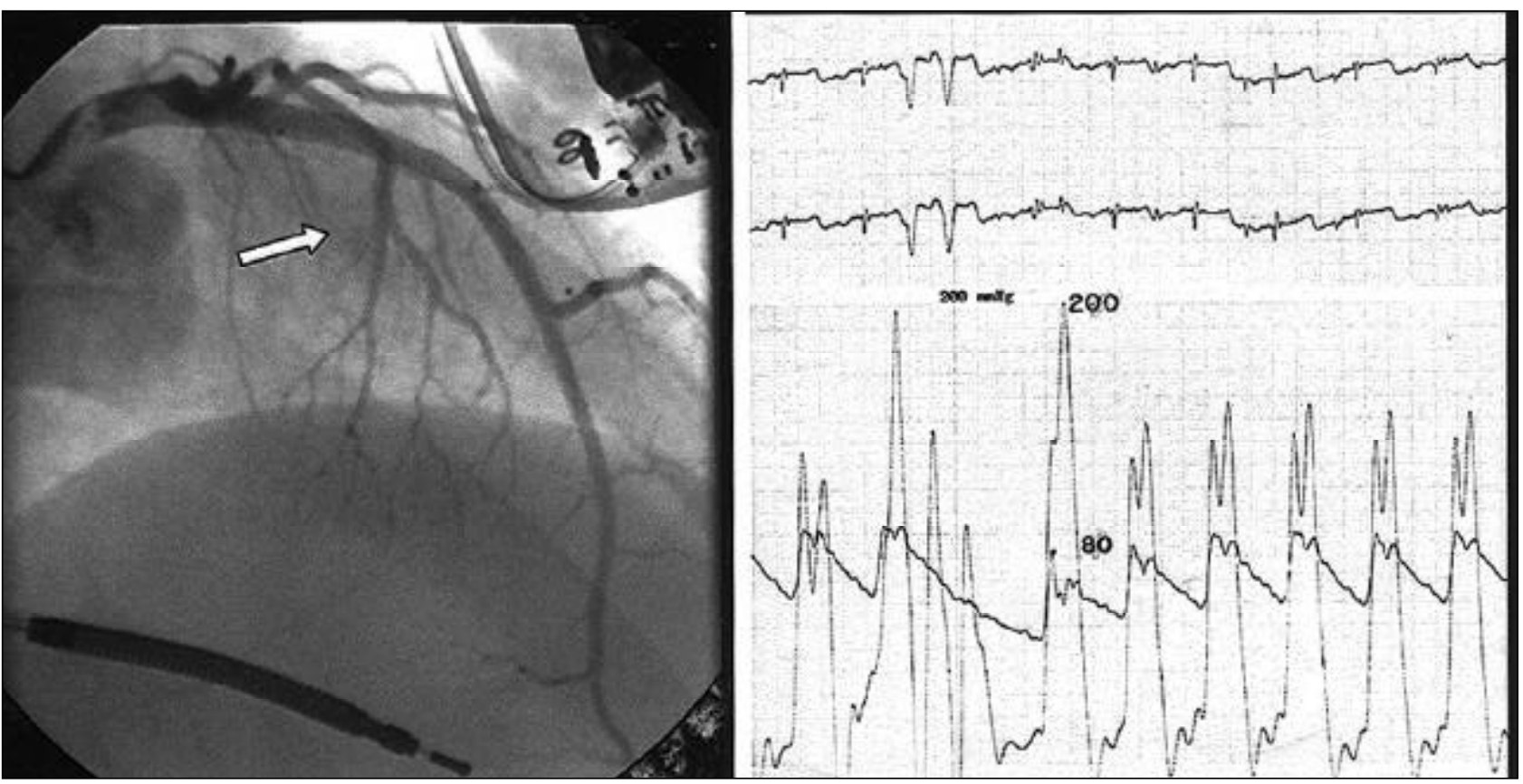

Fig. 1 - Opacification of the anterior interventricular artery in posteroanterior cranial projection. Note the importance of the first septal branch (arrow) to the left, and the presence of the cardioverter-defibrillator electrode at the right ventricular tip. On the right side, recording of the LV-AO simultaneous pressure is observed with an important intraventricular pressure gradient that increases after extrasystole. 


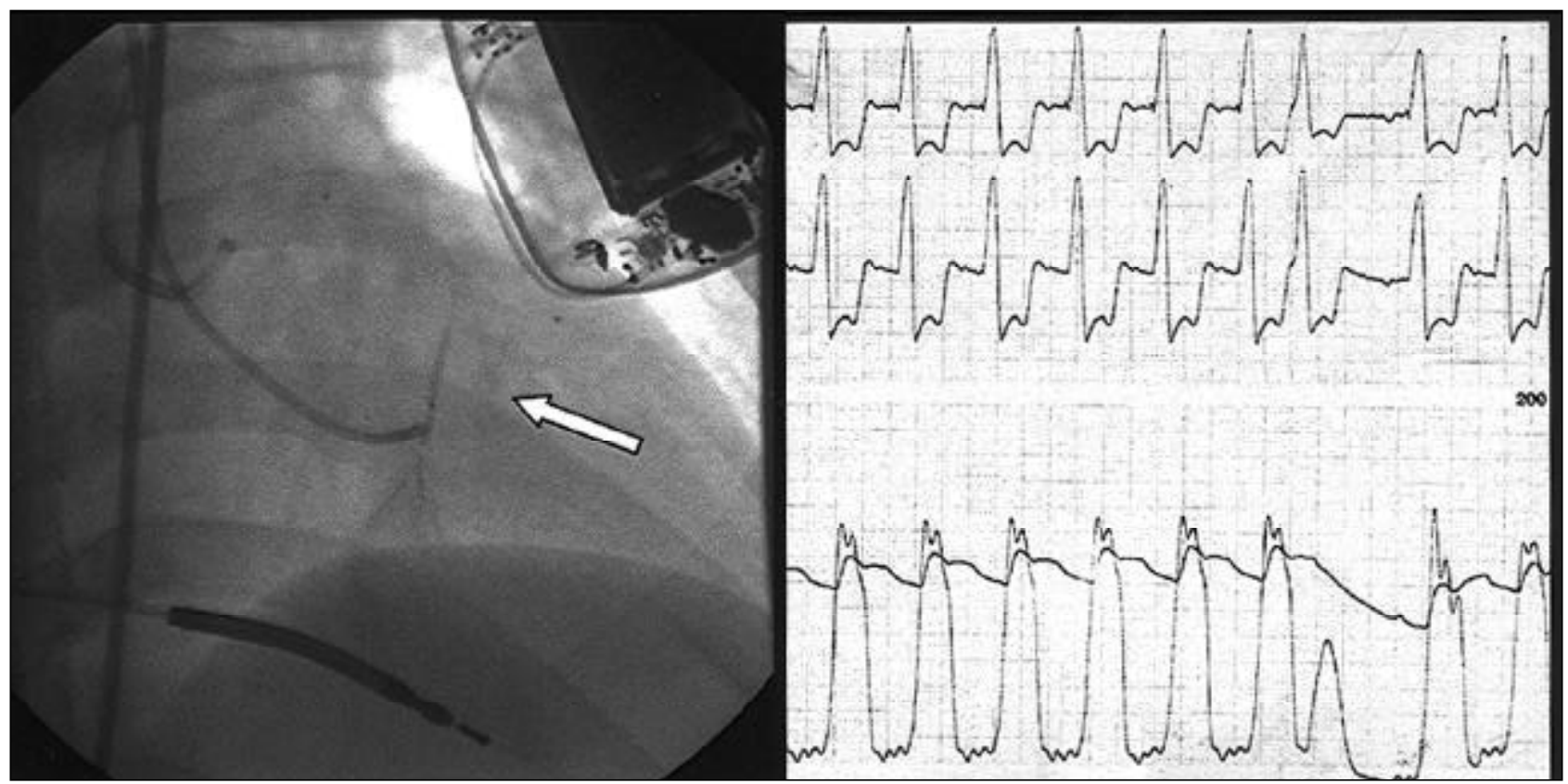

Fig. 2 - Image of the balloon inflated in the first septal branch chosen for the test. On the right side, the almost absent pressure gradient. With this result, continuation of the procedure is indicated.

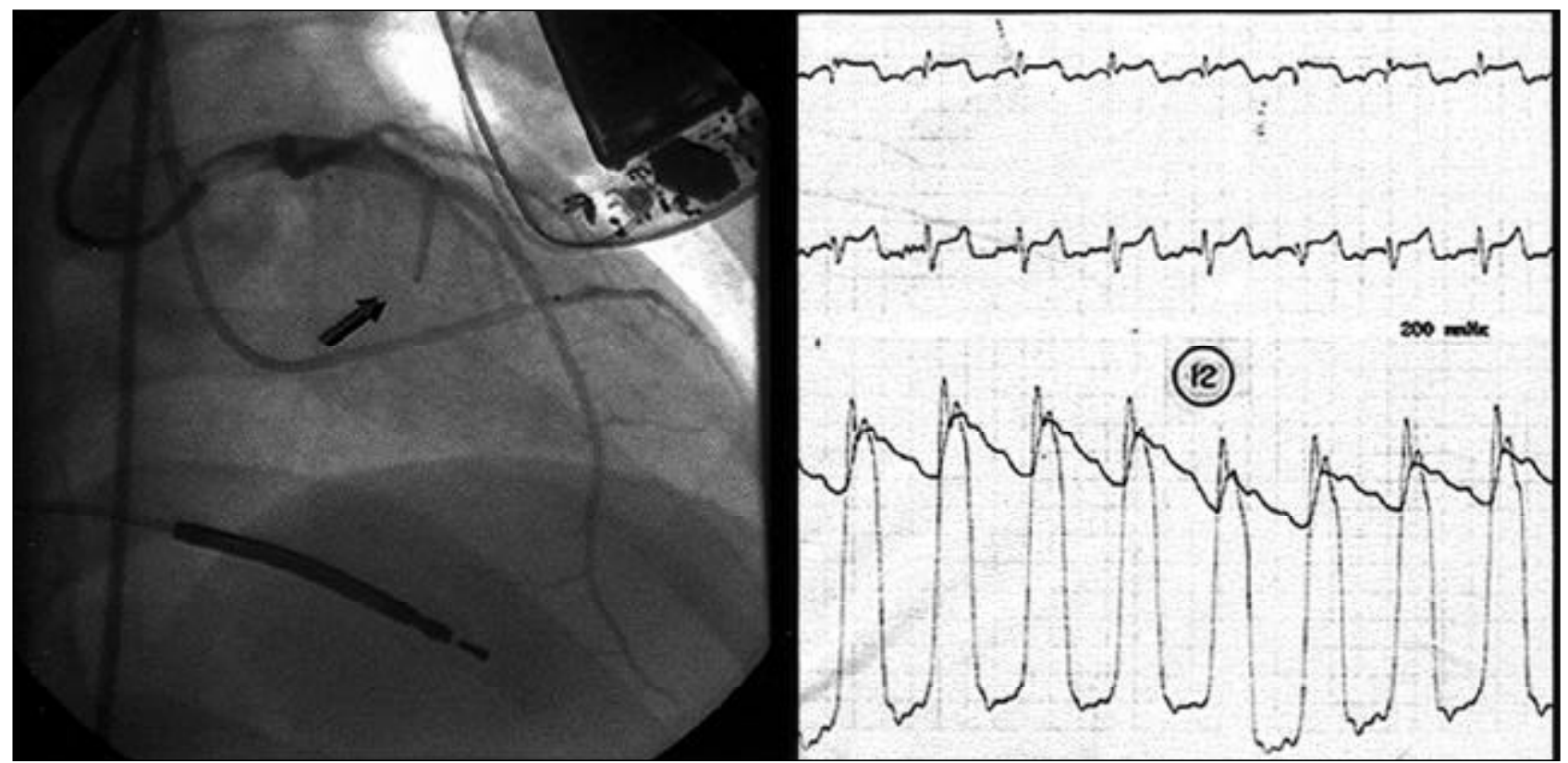

Fig. 3 - The last stage of the procedure. After alcohol infusion, amputation of the septal branch is seen on the left side, and nonexistence of pressure gradient on the right side.

branch. Two patients required occlusion of a second branch, because a significant pressure gradient persisted after occlusion of the first branch. Therefore, excluding these cases in which 2 branches underwent alcoholization, $3 \mathrm{~mL}$ of absolute alcohol was always injected during each septal alcoholization. The mean total duration of the procedure was $2 \pm 1$ hours, with a mean contrast medium consumption of $300 \pm 50 \mathrm{~mL}$.

Of the 18 patients who concluded alcoholization, complete abolition of the pressure gradient was observed in 10
$(55.5 \%)$ patients, even after extrasystole. In the 8 remaining patients, a basal residual gradient $<20 \mathrm{mmHg}$ was observed. In 1 patient with no gradient at rest and with a discrete gradient after extrasystole, intravenous dobutamine was used, which caused the severe intraventricular pressure gradient to reappear and be maintained, allowing the protocol to be followed. This patient, on the preceding day, had a peak gradient of $70 \mathrm{mmHg}$ on Doppler echocardiography at baseline.

Two patients with DDD-type pacemakers became refractory, in spite of taking full medication added to a beta- 
blocker and diuretics, and were referred for alcoholization. The procedures were successfully performed, and both patients became asymptomatic with a low dose of propranolol. The first patient did not require the pacemaker to be maintained due to the presence of sinus rhythm. This, however, did not occur to the second patient, who maintained the symptomatic total AV block with severe bradycardia (fig. 4).

Immediately before alcoholization, the data obtained with manometry in the catheterization laboratory showed a mean intraventricular pressure gradient of $67.63 \pm 24.25$ $\mathrm{mmHg}$ at baseline, and of $110.44 \pm 23.66 \mathrm{~mm} \mathrm{Hg}$ after extrasystole. After alcoholization, the intraventricular pressure gradient decreased to $3.81 \pm 1.90 \mathrm{mmHg}(\mathrm{t}=2.78 ; \mathrm{p}<0.05)$ at baseline, and to $9.56 \pm 2.63 \mathrm{mmHg}(\mathrm{t}=2.78 ; \mathrm{p}<0.05)$ after extrasystole. Doppler echocardiography performed prior to discharge from the intensive care unit (ICU) reproduced the results obtained in the catheterization laboratory, mainly in regard to peak gradient.

All patients had the most diverse arrhythmias in the catheterization laboratory (chart II). Ten $(55.5 \%)$ patients had acute complete atrioventricular block and severe bradycardia, requiring a transient pacemaker (fig. 5); 8 of them reverted to sinus rhythm until the second hour, and 2 required implantation of a definitive artificial cardiac pacemaker. One patient had ventricular fibrillation during alcohol injection, which was reverted with electric cardioversion (chart II).

All 18 patients undergoing the procedure and admitted to the ICU had significant elevations of the CK-MB enzymatic fraction (chart III).

Of the 22 patients initially referred for alcoholization of the septal artery and production of therapeutic septal infarction, 4 did not complete the procedure due to the following reasons: 3 patients had extreme tortuosity of the anterior interventricular artery, or thin septal branches, or tortuosity at the origin of the septal branch; the forth patient reported intense pain when entering the catheterization laboratory. The appearance of the left coronary artery on diagnostic coronary angiography suggested a diffuse spasm, which did not revert with local vasodilators, and the patient died after irreversible ventricular fibrillation.

In the ICU, all patients reported pain or a sensation of heaviness with a mean duration of $24 \pm 12$ hours. In a mean period of $4 \pm 1$ days, they were discharged asymptomatic, hemodynamically stable, and with normalized cardiac enzymatic levels. All remained on low dosages of beta-blockers

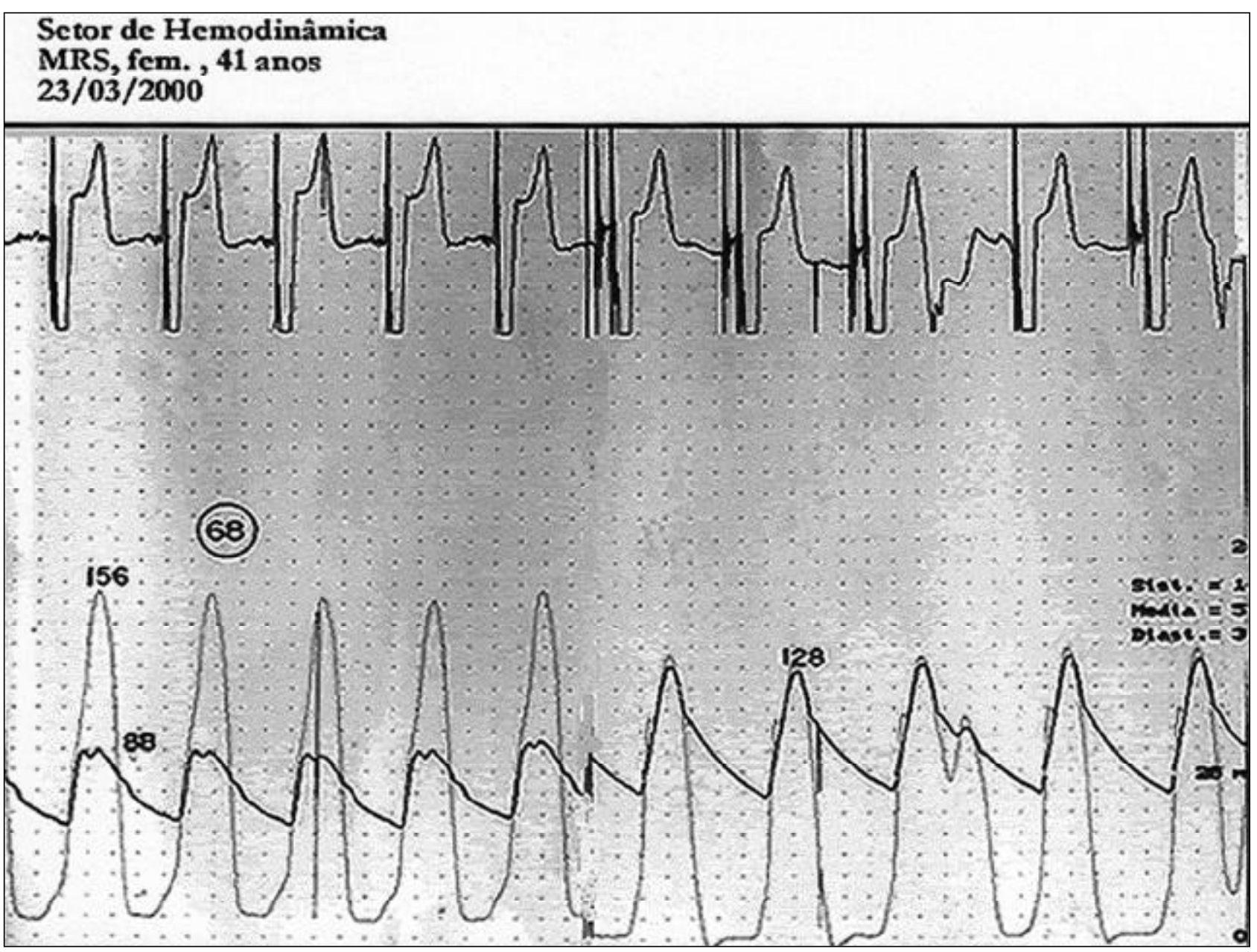

Fig. 4 - Pressure recording in a patient with definitive DDD-type pacemaker, before and after ablation with absolute alcohol. During follow-up, the need for maintaining the pacemaker was observed, due to persistence of symptomatic bradycardia. 


\begin{tabular}{|lrc|}
\hline \multicolumn{1}{|c|}{ Chart II - Complications } & \\
\hline - Failures & 4 & $(22.2 \%)$ \\
- Arrhythmias & & \\
Ventricular extrasystoles: & 18 & $(100 \%)$ \\
Supraventricular extrasystoles & 8 & $(44.4 \%)$ \\
Ventricular tachycardia & 12 & $(66.6 \%)$ \\
Supraventricular tachycardia & 4 & $(22.2 \%)$ \\
Sinus bradycardia & 8 & $(44.4 \%)$ \\
Ventricular fibrillation & 1 & $(5.5 \%)$ \\
Total AV block & 10 & $(55.5 \%)$ \\
$\quad$ Transient & 8 & $(44.4 \%)$ \\
$\quad$ Permanent & 2 & $(11.1 \%)$ \\
Complete right bundle-branch & & \\
block (CRBBB) & 10 & $(55.5 \%)$ \\
CRBBB + left anterior & 2 & $(11.1 \%)$ \\
hemiblock & & \\
Complete left bundle- & & \\
branch block & 1 & $(5.5 \%)$ \\
Left anterior hemiblock & 1 & $(5.5 \%)$ \\
- Pacemaker implantation & & \\
Transient & 1 & $(55.5 \%)$ \\
Definitive & 10 & $(11.1 \%)$ \\
Early & 2 & \\
Late & 1 & $(5.5 \%)$ \\
\hline
\end{tabular}

(propranolol at $40 \mathrm{mg} /$ day) after hospital discharge, which occurred in $10 \pm 2$ days. On ambulatory follow-up in the 6 th month, all patients were in NYHA functional class I.

The comparative analysis of the Doppler echocardiograms performed before alcoholization and on ambulatory follow-up showed a statistically significant reduction in the interventricular septal thickness, which passed from $20.3 \pm 0.32$ mm to $1.66 \pm 0.20 \mathrm{~mm}(\mathrm{t}=2.78 ; \mathrm{p}<0.05)$.

Another echo-Doppler variable that showed a statistically significant reduction was the peak pressure gradient, whose mean value passed from $90.69 \pm 23.47 \mathrm{mmHg}$ to $6.13 \pm 1.42 \mathrm{mmHg}(\mathrm{t}=2.78 ; \mathrm{p}<0.05)$. Some patients with residual peak gradient immediately after the procedure had a progressive reduction as time passed. The mean values of posterior wall thickness, left atrial diameter, and septum/ posterior wall ratio did not show statistically significant alterations.

The systolic anterior movement of the mitral valve (SAM), observed on Doppler echocardiography in all patients undergoing alcoholization, showed an extremely variable behavior during the echocardiographic assessment

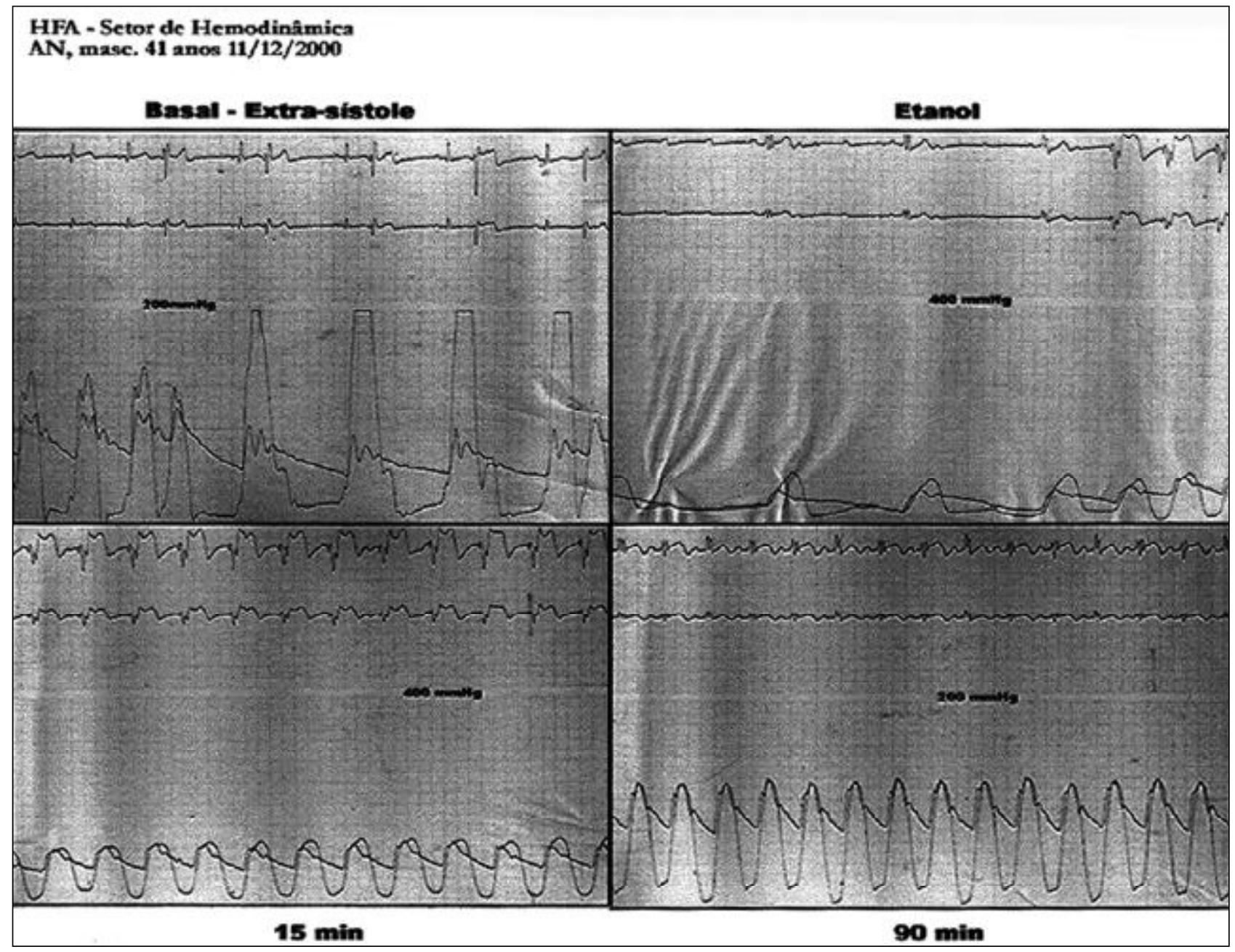

Fig. 5 - Patient with obstructive hypertrophic cardiomyopathy and a severe pressure gradient after extrasystole, which may be observed in the left upper quadrant. Intraseptal infusion of alcohol immediately induced total AVB followed by ventricular capture by an external pacemaker (right upper quadrant). In the right lower quadrant, equalization of the pressures and sinus rhythm can be observed. 


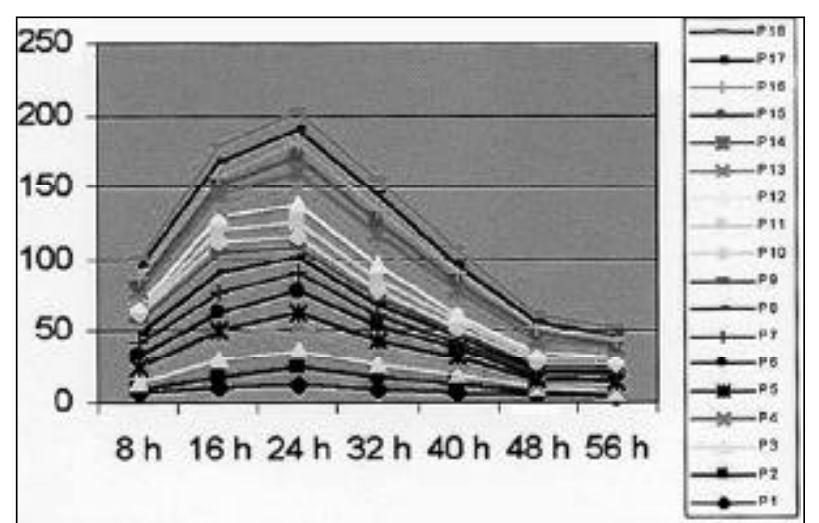

Chart III - Curve of the CK-MB enzymatic fraction in all patients undergoing septa alcoholization.

in the 6th month. In 12 patients, SAM showed a reduction in amplitude and duration; in 4, SAM disappeared completely; and in 2, SAM maintained the same pattern prior to the procedure.

From the electrocardiographic point of view, 6 months after the procedure, $12(66.6 \%)$ patients had complete right bundle-branch block, which was associated with anterosuperior fascicular block in 2 patients (fig. 6). One patient develo- ped isolated and complete left bundle-branch block (5.5\%), and another had isolated anterosuperior fascicular block.

In the period of $8 \pm 2$ months, $14(77.7 \%)$ patients remained in NYHA functional class I and 1 (5.5\%) in class II; in $3(16.6 \%)$, the symptoms relapsed and the intraventricular pressure gradient recurred in the ninth month. Two of these patients underwent a successful new alcoholization, returning to functional class I; the third patient, who refused any type of invasive treatment, remained in functional class II taking propranolol ( $240 \mathrm{mg} /$ day $)$ and diuretics.

\section{Discussion}

Alcoholization of the septal artery in hypertrophic obstructive cardiomyopathy acutely reduces interventricular septal thickness, with consequent widening of the left ventricular outflow tract and drop in the intraventricular pressure gradient. This occurs due to production of myocardial infarction circumscribed to the area irrigated by the occluded septal branch. Other acute beneficial hemodynamic effects were observed, such as those deriving from changes in the sequence of ventricular activation caused by alterations in its conduction system ${ }^{42,44}$. An even greater reduction in the intraventricular gradient due to ventricular

\section{Instituto do Coração de Taguatinga - DF JPJ, 32a \\ $12 / 06 / 98$}

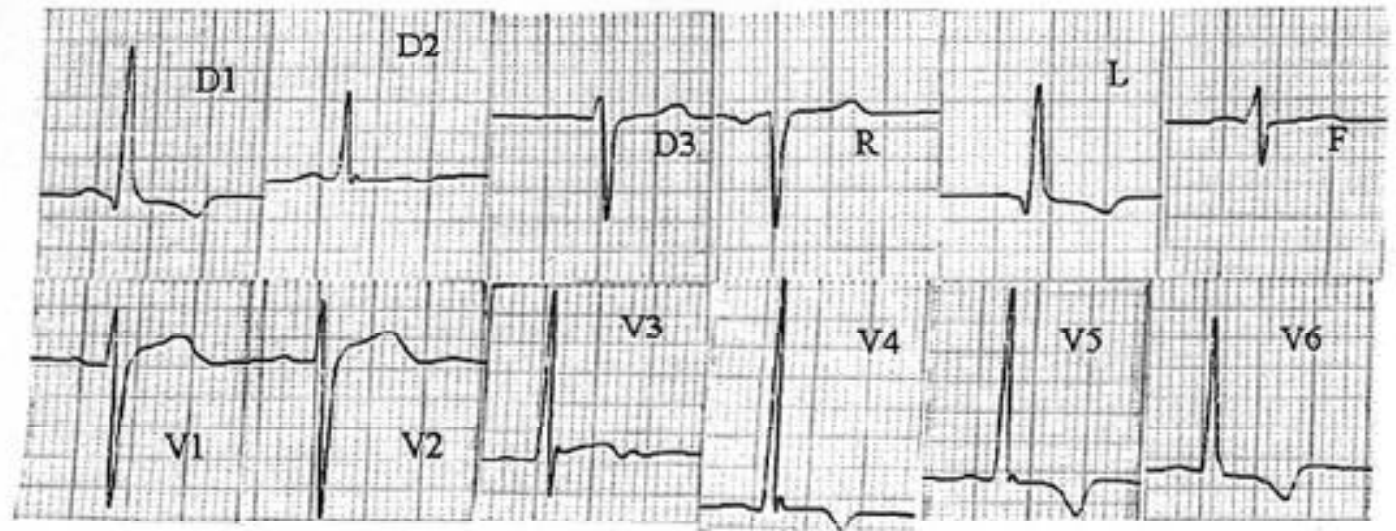

$17 / 12 / 99$

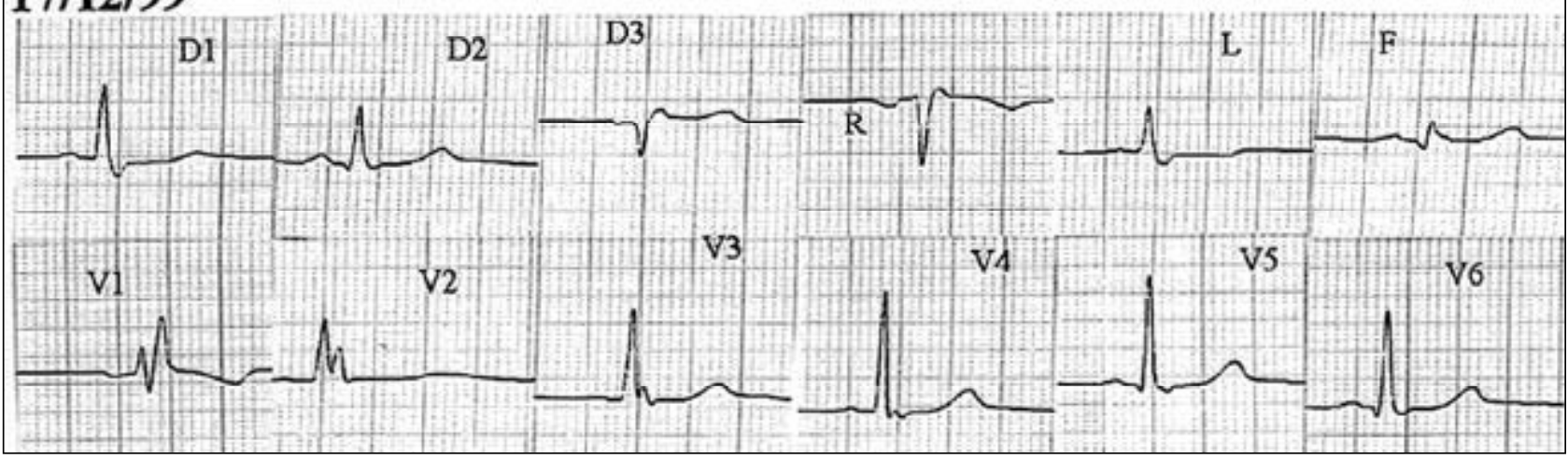

Fig. 6 - Electrocardiogram before and late after septal alcoholization showing development of right bundle-branch block. Note the appearance of small "Q waves" in the anterolateral wall. 
remodeling and an increase, although not significant, in its diastolic diameter were observed as late results of septal infarction ${ }^{41,47,50}$. The ventricular posterior wall, many times exhibiting a certain degree of secondary hypertrophy, may also show some degree of involution due to a reduction in cavitary pressure according to some reports ${ }^{41,47,48}$. The summation of these factors could lead to a reduction in the degree of diastolic heart failure, and, consequently, to a significant improvement in the patients' functional class.

In many individuals, hypertrophy is initially located in the interventricular septum. From then on, as the obstruction progresses and the intraventricular pressure increases, a compensatory concentric hypertrophy gradually develops. This secondary hypertrophy is almost always associated with fibrosis that may ultimately lead to heart failure, ventricular arrhythmias, and sudden death. Therefore, an early interruption of the process in the course of hypertrophic obstructive cardiomyopathy may prevent or attenuate the development of hypertrophy and fibrosis and their subsequent complications, including sudden death ${ }^{50,53}$.

A significant advantage of alcoholization as compared with that of septal reduction by conventional surgery is that its therapeutic effect is predictable; the nonreduction in the intraventricular pressure gradient with transient occlusion of the septal branch with the balloon contraindicates its alcoholization. The surgical treatment, even performed in referral centers, has a significant mortality rate and its percentage of failure ranges from $10 \%$ to $30 \%{ }^{27-35,51}$. Another advantage is that alcoholization may be repeated later.

Compared with DDD-type pacemakers, septal alcoholization causes a reduction in septal thickness with all its subsequent beneficial effects, which do not occur with the electric treatment. The symptomatic improvement determined by pacemakers is currently known to be more consistent with a substantial placebo effect ${ }^{24,26}$. In most of these patients, a mild reduction in left ventricular outflow tract gradient was observed and only a few patients older than 65 years and at a high risk for surgery or septal alcoholization actually benefited from that type of treatment.

Percutaneous transluminal septal alcoholization is indicated in symptomatic patients in functional class III or IV, refractory to thorough medicamentous treatment or those who have important side effects due to the use of those medications. Asymptomatic or mildly symptomatic patients meet the inclusion criteria as long as they have documented risk factors for sudden death. Patients with hypertrophic obstructive cardiomyopathy, previously myectomized or with DDD-type pacemakers, or both, may also undergo percutaneous transluminal septal alcoholization. The procedure is only contraindicated in patients with concomitant heart disease indicated for surgical treatment.

Up to the present moment, publications about percutaneous transluminal septal alcoholization or ablation are scarce. In 2000, Kuhn et al ${ }^{50}$ estimated that only 1,000 patients in the entire world had undergone that treatment. The immediate results originating from several international centers have accounted for the high percentage of success ${ }^{41,42,45,51,52}$. It was also observed that, in most of these centers, to reach a significant reduction in the pressure gradient, occlusion of several septal branches was required, which differs from the present case series.

The Brazilian experience is still limited with only 3 studies published, of which 2 are case reports and the other comprises a series of 6 cases ${ }^{39,43,55}$. However, all reports confirmed the efficacy of the method in the treatment of hypertrophic obstructive cardiomyopathy.

In regard to acute complications of septal alcoholization, a universal consensus exists regarding the care and attention that should be paid to the possible complications of myocardial infarction, even in the one induced at the hospital with all available resources. As observed in this case series, severe arrhythmias, such as ventricular or supraventricular tachyarrhythmias frequently occur (chart II). Likewise, atrioventricular blocks of greater or lesser degree may occur depending on the location and type of supply to the atrioventricular node. Similarly to surgical myectomy, although with no report in the literature, iatrogenic perforation of the interventricular septum (ventricular septal defect - VSD) may occur, because a significant thinning of the infarcted region nourished by the septal branch that underwent alcoholization occurs. Another possible mechanical complication is acute mitral insufficiency due to rupture of the papillary muscle depending on the distribution pattern of the alcohol infused. Finally, cerebral embolism present in a small number of cases of myectomy may also occur in alcoholization.

One of the most feared complications of septal ablation is hospital mortality, which is around $5 \%$ in the centers of greater experience. The causes cited are as follows: cerebral and coronary embolism, unexpected trifascicular block of the His bundle, pericardial tamponade due to cardiac perforation by a pacemaker's thread, pulmonary embolism, and extensive myocardial infarction due to alcohol reflux to the anterior interventricular artery ${ }^{53}$. In the present study, of the 22 patients who underwent septal ablation, 1 (4.5\%) hospital death occurred and was attributed to diffuse coronary spasm, which occurred before the beginning of the procedure.

In our case series, the following complications were not observed: cerebral embolism, structural complication of the VSD type, acute mitral insufficiency, and pulmonary embolism.

In regard to the late follow-up of patients undergoing septal ablation, the literature reports up to 5 years of observation ${ }^{38,44,45,51,53}$. The incidence of late cardiac complications was low, but not insignificant. In regard to sudden death, the incidence reported in the literature is below $5 \%$. In our case series, 1 late death occurred at the end of 9 months (5.5\% of the total). It was the sudden death of a patient, who at the 6-month follow-up was in functional class I, exclusively using $40 \mathrm{mg} /$ day of propranolol. However, this fact may suggest the need for using cardioverter-defibrillator in patients, who, although asymptomatic after successful alcoholization, have a documented high risk for that complication. 
The incidence of late complications and severe arrhythmias is extremely reduced. In regard to these facts, it is a consensus among authors to recognize that the number of cases investigated and the period of observation have not been sufficient for a definitive judgment. A fact that is very clear, however, is the progressive reduction in symptomatology in the late follow-up, when most patients remain in functional class I and with a good response to exercise testing.

Data in the literature have also shown that, in regard to the intraventricular pressure gradient, the follow-up of large groups of patients has always shown the maintenance of the early results and even a progressive reduction or disappearance of the residual gradients, which may mean a response to late ventricular remodeling, as occurs in many cases of myocardial infarction.

In all case series reported, the late findings on Doppler echocardiography have shown a significant reduction in the thickness of the interventricular septum and of the left ventricular posterior wall, indicating a late beneficial response to the reduction in the resistance to ventricular emptying.

Finally, another interesting point is that, despite success in removing the obstruction of the left ventricular subaortic region, ventricular diastolic dysfunction is 1 of the major components of the primary disease. Therefore, the recommendation is to permanently maintain drug therapy based on low dosages of beta-blockers.

The long-term results of the procedure involving a large case series are still unknown. However, according to the history of medicine, most therapeutic procedures established for late assessment passed by results at the short- and midterm that were as good as those shown for this method.

Septal alcoholization caused a beneficial effect, which may be the summation of the following effects of surgical and electrical treatment: widening of left ventricular outflow tract, ventricular remodeling due to infarction, and altered ventricular activation. Another significant advantage of septal alcoholization is the possibility of being easily repeated at any time, mainly in the recurrence of intraventricular pressure gradient and in myectomized patients with poor surgical results.

The major complication of septal alcoholization is the permanent total atrioventricular block with the indication for definitive pacemaker implantation. However, long-term observations and comparative studies with other invasive therapies involving large case series are required to consolidate percutaneous transluminal septal ablation among the cardiological therapeutic tools.

In conclusion, based on data obtained in the present series, percutaneous transluminal septal alcoholization used to treat patients with refractory hypertrophic obstructive cardiomyopathy has proved efficient due to the 2 following facts: 1) occurrence of an immediate and significant reduction in the intraventricular pressure gradient in baseline conditions and after extrasystole; 2) the technique proved to be reproducible with a minimum of immediate morbidity. The need for definitive pacemaker implantation was the most frequent acute complication and recurrence of the symptoms and reappearance of the pressure gradient occurred in the midterm. The late mortality rate due to sudden death was low, but not insignificant, suggesting the need for implanting a defibrillator-cardioverter in patients at risk for sudden death. Unfavorable coronary anatomy due to tortuosity was the major factor responsible for the unsuccessful cases.

However, percutaneous transluminal septal alcoholization has not yet been considered as the first-choice treatment for this modality of hypertrophic cardiomyopathy. Probably, a broader randomized study comparing percutaneous transluminal septal alcoholization with surgical treatment may provide a better judgment of the actual benefit of the method.

\section{References}

1. Maron BJ, Bonow RD, Canon RD, Leon MB, Epstein SE. Hypertrophic cardiomyopathy. N Engl J Med 1987; 316: 780-9.

2. Davies MJ, MCKenna WJ. Hypertrophic cardiomyopathy: an introduction to pathology and pathogenesis. Br Heart J 1994; 72(suppl): S2-S3.

3. Marian AJ, Roberts R. Recents advances in the molecular genetics of hypertrophic cardiomyopathy. Circulation 1995; 92: 1336-47.

4. Spirito P, Seidman CE, MCKenna WJ, Maron JB. The management of hypertrophic cardiomiopathy. N Engl J Med 1997; 33: 775-85.

5. Maron BJ, Gardin JM, Flack JM, Gidding SS, Kurusaki TT, Bild DE. Prevalence of hypertrophic cardiomyopathy in a general population of young adults: echocardiographic analysis of subjects in the Cardiac Study. Circulation 1995; 92: 785-9.

6. Watkins $\mathrm{H}$. Multiple disease genes cause hypertrophic cardiomyopathy. $\mathrm{Br}$ Heart J 1994; 72(suppl): S4-S9.

7. Moolman JC, Corfield VA, Posen B, Ngubela K, Seidman C, Brink P. Sudden death due to troponin T mutations. J Am Coll Cardiol 1997; 29: 549-55.

8. Arteaga-Fernandez E. Cardiomiopatia hipertrófica - Estudo da sobrevida e de fatores prognósticos. (Tese de Doutorado) Faculdade de Medicina da USP. São Paulo, 1998.

9. Maron BJ, Casey AS, Poliac LC, Gohman TE, Almquist AK. Clinical course of hypertrophic cardiomyopathy in a regional United States cohort. JAMA 1999; 281: $650-5$.
10. Abbasi AS, MCAlpin RN, Eber LM, Pearce ML. Echocardiographic diagnosis of idiopathic hypertrophic cardiomyopathy without outflow obstruction. Circulation 1972; 46: 897-904.

11. Jiang L, Levine RA, King ME, Weyman AE. An integrated mechanism for systolic anterior motion of the mitral valve in hypertrophic cardiomyopathy based on echocardiographic observations. Am Heart J 1987; 113: 633-44.

12. Wigle EP, Sanson Z, Henderson M, Ruddy T, Fulop J, Rakowisky H. Hypertrophic cardiomyopathy: the importance of the site and extent of hypertrophy: a review. Prog Cardiovasc Dis 1986; 28: 1-83.

13. Blanchard DG, Ross J. Jr. Hypertrophic cardiomyopathy: prognosis with medical or surgical therapy. Clin Cardiol 1991; 14: 11-9.

14. Rosing D, Keny K, Bores J, Maron BJ, Epstein SE. Verapaniil therapy: a new approach to the pharmacologic treatment of hypertrophic cardiomyopathy. 1Hemodynamic effects. Circulation 1979; 60: 1201-8.

15. Pollick C, Gershony G, Szarga C, Wogle ED. Dysopiramide: hemodynamic effects in hypertrophic cardiomyopathy (abstract). Circulation 1983; 68(suppl. 3): 161 .

16. Anderson D, Ralff G, Ports T, Brundage B, Parmley W, Chaterjee K. Hypertrophic obstructive cardiomyopathy. Effects ofacute and chronic verapamil treatment on left ventricular systolic and diastolic function. Br Heart J 1984; 51: 523-9.

17. Spirito P, Maron BJ, Bonow RO, Epstein SE. Occurrence and significance of 
progressive left ventricular wall thinning and relative cavity dilatation in patients with hypertrophic cardiomyopathy. Am J Cardiol 1987; 60: 123-9.

18. MCKenna WJ, Harris L, Rowland E. Amiodarone for long-term management of patients with hypertrophic cardiomyopathy. Br Heart J 1985; 53: 412-6.

19. Fannanapazir L, Leon MB, Bonow RO, Tracy CM, Cannon RO, Epstein SE Sudden death during empiric amiodarone therapy in symptomatic hypertrophic cardiomyopathy. Am J Cardiol 1991; 67: 169-74.

20. Wigle ED, Rakowsky H, Kimballi BP, Williams WG. Hypertrophic Cardiomyopathy: clinical spectrum and treatment. Circulation 1995; 92: 1680-92.

21. Jeanrenaud X, Goy JJ, Kappenberger I. Effects of dual-chamber pacing in hypertrophic obstructive myocardiopathy. Lancet 1992; 339: 1318-23.

22. Fananapazir L, Epstein ND, Curiel RV. Long-term results of dual chamber (DDD) pacing in obstructive hypertrophic cardiomyopathy: evidence for progressive symptomatic and hemodynamic improvment and reduction of left ventricular hypertrophy. Circulation 1994; 90: 2731-42.

23. Kappenberger I, Linde C, Daubert C. Pacing for obstructive hypertrophic cardiomyopathy. Eur Heart J 1997; 18: 1249-56.

24. Nishimura RA, Hayes DL, Ilstrup DM. Effect of dual-chamber pacing on systolic and diastolic function in patients with hypertrophic cardiomyopathy: acute doppler echocardiographic and catheterization hemodynamic study. J Am Coll Cardiol 1996; 27: 421-30.

25. Nishimura RA, Trusty JM, Hayes DL. Dual-chamber pacing for hypertrophic cardiomyopathy: a randomized, double blind, crossover trial. J Am Coll Cardiol 1997; 29: 435-41.

26. Maron BJ, Nishimura RA, MCKenna WJ, Rakowsky H. Assessment of permanent dual-chamber pacing as a treatment for drug-refractory symptomatic patients with obstructive hypertrophic cardiomyopathy (The M-Pathy Study). Circulation 1999; 99: 2927-33.

27. Morrow AG, Reitz BA, Epstein SE. Operative treatment in hypertrophic subaortic stenosis: techniques and the resultsof pre and postoperative assessments in 83 patients. Circulation 1975; 52: 88-102.

28. Seiler C, Hesse OM, Schoenbeck M. Long-term follow-up of medical versus surgical therapy for hypertrophic cardiomyopathy: a retrospective study. J Am Coll Cardiol 1991; 17: 634-42.

29. MCCully RB, Nishimura RA, Rajik AJ. Extent of clinical improvement after surgical treatment of hypertrophic obstructive cardiomyopathy. Circulation 1996; 94: 467-71.

30. MCintosh CL, Maron BJ. Current operative treatment of obstructive hypertrophic cardiomyopathy. Circulation 1988; 78: 487-95.

31. Mohr R, Schaff HV, Danielson GK, Puga FJ, Pluth IR, Tajik AJ. The outcome of surgical treatment of hypertrophic obstructive myocardiopathy: experience over 15 years. J Thorac Cardiovasc Surg 1989; 97: 666-74.

32. Chahine RA. Surgical versus medical treatment of hypertrophic cardiomyopathy: is the perspective changing? J Am Coll Cardiol 1991; 17: 643-5.

33. Cooley DA, Leachman RD, Wukach DC. Difuse muscular subaortic stenosis: surgical treatment. Am J Cardiol 1973; 31: 1-6.

34. MCintosh CL, Maron JB. Current operative treatment of obstructive hypertrophic cardiomyopathy. Circulation 1988; 78: 487-95.

35. Krajcer Z, Leachman R, Cooley DA, Coronado R. Septal myotomy-miectomy versus mitral valve replacement in hypertrophic cardiomyopathy: ten year follow-up in 185 patients. Circulation 1989; 80(suppl. I): 57-64.

36. Sigwart Y. Non surgical myocardial reduction for hypertrophic obstructive cardiomyopathy. Lancet 1995; 346: 211-4.
37. Kuhn H, Gietzen F, Lenner CH, Gerenkamp T. Induction of subaortic septal ischemia to reduce obstruction in hypertrophic obstructive cardiomyopathy. Eur Heart J 1997; 18: 846-51.

38. Knight C, Kurbaan A, Seggewiss U, Henein M, Gunning M, Harrington D. Non surgical septal reduction for hypertrophic obstructive cardiomyopathy. Circulation 1997; 95: 2075-81.

39. Marin Neto JA, Lima Filho MO, Schumidt A, Maciel BC. Cardiomiopatia hipertrófica tratada por técnica intervencionista de redução septal. Arq Bras Cardiol 1998; 70: 343-50.

40. Seggewiss H, Faber L, Gleichmann U. Percutaneous transluminal septal ablation in hypertrophic obstructive cardiomyopathy. Thorac Cardiovasc Surg 1999; 47: 84-100.

41. Colombo A, Airoldi F, Di Mario C, Catanoso A, Tolaro S. Progressive decrease of outflow gradient and septum thickness after percutaneous alcoholization of interventricular septum in hypertrophic obstructive cardiomyopathy. Ital Heart J 2000; 1: 200-6.

42. Oomann A, Ramachandran P, Osmann M. Percutaneous transluminal septal myocardial ablation in drug-resistant hypertrophic obstructive cardiomyopathy. J Invasive Cardiol 2001; 13: 526-30.

43. Esteves MAB, De Matos RB, Lima Filho A. Oclusão da artéria septal na cardiomiopatia hipertrófica obstrutiva. Rev Bras Cardiol Invas 2001; 9: 20-3.

44. Kuhn H, Gietzen F, Schafers M. Catheter interventional therapy of hypertrophic obstructive cardiomyopathy by transcoronary ablation of septum hypertrophy: changes of subaortic septum. Eur Heart J 1997; 18(suppl): 605-8.

45. Gleichmann U, Seggewiss H, Faber L. Kathetertherapie der hypertrophen obstruktiven Kardiomyopathie. Detsch Med Wochenschr 1996; 21: 679-85.

47. Faber L, Meissner A, Ziemsen P. Percutaneous transluminal septal myocardial ablation for hypertrophic obstructive cardiomyopathy: long-term follow-up of the first series of 25 patients. Heart 2000; 83: 326-31.

48. Lakkis NM, Nagueh SF, Dunn JK. Nonsurgical septal reduction therapy for hypertrophic obstructive cardiomyopathy: one-year follow-up. J Am Coll Cardiol 2000; 36: 852-5.

49. Spirito P, Rapezzi C, Autuore C. Prognosis of asymptomatic patients with hypertrophic cardiomyopathy and nonsustained ventricular tachycardia. Circulation 1994; 90: 2743-7.

50. Kuhn H. Transcoronary ablation of septal hypertrophy (TASH): a 5-year experience. J Cardiol 2000; 89: 559-64.

51. Sherif FN, Steve RO, Nasser ML, et al. Comparison of ethanol septal reduction therapy with surgical myectomy for the treatment of hypertrophic obstructive cardiomyopathy. J Am Coll Cardiol 2001; 38: 1701-6.

52. Kurbaan AS, Seggewiss H, Henein MY. Non-surgical septal reduction for hypertrophic obstructive cardiomyopathy: long term follow-up of the early patients. Circulation 1997; 96(suppl): 2587A.

53. Gietzen F. Acute and long term results after transcoronary ablation of septal hypertrophy (TASH), catheter interventional treatment for hypertrophic cardiomyopathy. Eur Heart J 1999; 20: 1342-54.

54. Cano MN, Cano SJF, Oliveira DC, et al. Ablação percutânea da artéria septal no tratamento da cardiomiopatia hipertrófica. Rev Bras Cardiol Invas 2001; 9: 13-8.

55. Conselho Nacional de Saúde. Resolução No 196 de 10 de Outubro de 1996. In: Diretrizes éticas internacionais para pesquisas biomédicas envolvendo seres humanos. Bioética 1996; 3: 95-126. 